\title{
Research on the Presence of Country and Changes of Yiyang
}

\author{
Shi Lei \\ Jiangxi Science \& Technology Normal University, Music Academy, Nanchang, Jiangxi, 330013
}

\author{
Keywords: The Presence of the Country, Yiyang Cavity, Change
}

\begin{abstract}
This paper through the traditional methods of historical texts and oral history, showing the last century since 50s with the establishment of national political power. The will of the government in the light of its general trend entered into drama and traditional music culture, thus changing the face of survival and development path of the Yiyang chamber of this kind of intangible cultural heritage. I choose "Yiyang cavity" as a starting point, from the inheritance of Yiyang cavity predicament, connection, again interrupt and start with the reconstruction, explore the presence of national drama heritage, under the leadership of the government.
\end{abstract}

\section{The History of Yiyang Cavity and the Plight of Inheritance Before 1949}

Yiyang cavity originated in Jiangxi Yiyang in the history of ancient Chinese extremely brilliant, ranked four tune. It was because of the "noise" and "misuse of Xiang language", sung by one person, all chimed in, "and other characteristics have very big development. There are a lot of records of the Yiyang chamber, which Tang Xianzu in "Yihuang County Qingyuan" drama God Temple (1602) is described on the singing characteristics: "the river west of Yiyang, the festival to the noisy drum". This reflects its distinctive features, with orchestral percussion accompaniment, the noise and misuse of Xiang language one sings and singing, singing with rolling. However, after entering the middle of the Qing Dynasty, Yiyang cavity is caught in a dilemma: the tanqiang, such as Kunqiang tune system (especially the tanqiang) in Jiangxi show in gradually increasing, at the same time, the local tea nanbeici opera gradually rise, more and people love this old tune less, Yiyang cavity the performance, social class, the actors were gradually decreased [1-2]. To the Qing Qianlong and the records of the Yiyang cavity and its class society, it is difficult to find the historical materials about the Yiyang cavity after Qianlong. An analysis of three hundred kinds of historical materials in Yiyang

In the twenty-four years of the Republic of China (1935), the last speech dedicated Yiyang cavity of Ming Qing class to sing music Luantan cavity. At that time, most of the theater, is in Yiyang, one of the cavity Kunqiang, strum - tune board, but Yiyang is still in the cavity around, its excellent voice script have been lost, can sing Yiyang cavity actor also less and less [3]. Many Yiyang cavity actor lost troupe, had to perform in a cavity Luantan troupe. To the early days of liberation, there is no Yiyang chamber troupe, Yiyang chamber artists only all exist, and the situation is worrying. Wang Shiren, the Yiyang musician in the Republic of China, had described this:

To the beginning of the liberation, there is no special singing power team; nor a complete Gaoqiang opera, only 03 of the remaining four drop us a few pitched singing old man, dispersed in the Luantan class, every day for daily necessities to worry about, not liberation, we these old bones do not know throw in what place; what is art [4].

However, the Yiyang tune not only fell into the situation of no class society, but also the artists could not survive. The singing of their operas gradually shrank under such difficult circumstances, and the number of them came down less and less. Li Nanshui, an artist of Yiyang, who was born in Guangxu years, described this situation as follows:

We have 18 copies of the original high pitched drama, by the time I get to work, only 13 of the 13, this is the most I Wang Yufa taught. When my Master Li Jia Fu taught me, it was only 10. My three years of apprenticeship, and becomes the 8 . When I was 30 years old, I did have 7, and it was still very incomplete. At the beginning of the liberation, there is no show, only occasionally can see several single fold Luantan in high pitched drama class. Some of the wonderful performing arts of 
the older generation were also lost.

In this era, if there is no intervention of the national government, only relying on folk power or people's aesthetic preferences, the Yiyang chamber is basically close to extinction. In the 1950-1960 years, due to the intervention of the government, the Yiyang tradition, which was about to be interrupted and came up again.

\section{The Intervention of the Government Made the Yiyang Cavity "Back to Life" (1949-1966)}

At the beginning of the founding of the PRC, in order to bring opera into the whole national system, in 1951, the central government of the Communist Party of China issued a directive on the reform of operas, with the main contents of "changing people, restructuring and changing drama". In the past ten years, the government has implemented a series of reform policies of the folk opera opera, were planned, a wide range of collection and transmission. Yiyang cavity also catch a ride this wave of reform, even at the brink of lost position, or be handed down. In 1951, Shi Linghe, the current playwright of the Jiangxi Provincial Federation of Literary Federation, began the rescue work in Yiyang. The specific implementation of the rescue work is the relevant personnel of the Jiangxi Provincial Cultural Federation and the Jiangxi Provincial Cultural Bureau [5-6]. They began to organize rescue Yiyang cavity for cavity script, Yiyang old artist, recording music tunes.

In October 1953, the Jiangxi Provincial Cultural Bureau held a well-known "Jiangxi class" is the first Yiyang cavity actor training class, the number of Yiyang artists from Jingdezhen, Poyang chamber, Leping and other places, the capital of Nanchang, teaching classes. There are 30 students were the first Yiyang cavity actor training, most of them become the representative of the inheritance and development in Jiangxi later in Yiyang cavity.

Shi Linghe said this was the Yiyang cavity I saw when I was young, and then he gave a report to the first Jiangxi provincial governor of the year called Shao Shiping. Shao Shiping is very happy to hear, Shao Shiping, he himself is Yiyang, so he (later called Jiangxi Jiangxi was called Yiyang Raohe tune) cavity super happy, he said, "we must tap the (Yiyang cavity), basically can not see". So he sent a text, in 1953, to let the Culture Bureau at the time to build "Yiyang opera training class", to train new successors [7-8].

The behavior of the government of Yiyang actor training based on the cavity cavity Yiyang endangered once again handed down, and the inheritance of a more complete, not only outfit complete, and tune repertoire, and the performances are all got a more comprehensive inheritance.

\subsection{The inheritance of the "nine lines" in the Yiyang chamber}

Table 1. Yiyang chamber when "nine line head" and successors

\begin{tabular}{|c|c|c|c|}
\hline & $\begin{array}{l}\text { Business } \\
\text { name }\end{array}$ & Inheritor's name & $\begin{array}{c}\text { Inheritor life and } \\
\text { death time }\end{array}$ \\
\hline \multirow{3}{*}{$\begin{array}{l}\text { Third } \\
\text { flower }\end{array}$} & Big flower & Li Fudong & 1894-1962 \\
\hline & $\begin{array}{l}\text { Second } \\
\text { flower }\end{array}$ & Ye Sanyi & 1881-1967 \\
\hline & $\begin{array}{l}\text { Third } \\
\text { flower }\end{array}$ & Xu Shuanglin & 1886-1959 \\
\hline \multirow{3}{*}{$\begin{array}{l}\text { San } \\
\text { Sheng }\end{array}$} & $\begin{array}{c}\text { Old } \\
\text { students }\end{array}$ & Xu Weidong & 1894-1964 \\
\hline & $\begin{array}{l}\text { Main male } \\
\text { role }\end{array}$ & Li Nanshui & 1894-1972 \\
\hline & Niche & Zheng Ruisheng & $1905-1966$ \\
\hline \multirow{3}{*}{$\begin{array}{l}\text { San } \\
\text { Dan }\end{array}$} & Zheng Dan & Yu Liuxi & 1889-1954 \\
\hline & Xiao Dan & Feng Yijin & $1895-1973$ \\
\hline & Lao Dan & Gong Qinquan & 1887-1961 \\
\hline
\end{tabular}


First of all, Yiyang Yiyang chamber cavity actor training to teach singing master just nine people (they are born in the Qing Dynasty, the old artist Yiyang accent) nine singing master and musician Wang Shiren and drummer Xia Yichang nine to complete the Yiyang business chamber passed down: three, three, three Dan flowers.

\subsection{The Yiyang chamber music repertoire and tunes}

As mentioned above, although the play of Yiyang opera has been much less than those of the predecessors in the nine masters, their Yiyang Opera plays have been preserved. During this period the actor training class, these old artists and musicians together with wire recorder to record 10 plays and more than 200 tune, and at the end of 1950s with musical notation recorded. These numbered records have been preserved to this day, but what a wire recorder recorded the repertoire and tune but due to improper storage (or not enough attention?) And it was damaged.

\subsection{The inheritance of the Yiyang Opera}

The performance of the Yiyang cavity is a complete and comprehensive system. In this government - led inheritance in 50s, the performance of the Yiyang chamber was inherited in a more complete way. First of all, the basic work is divided into "four five". "Four" are singing, acting, dancing, reading skills, and the "Five" for the "eye body steps", including "pull top, waist, legs, garden field, walk, turn, flutter and fall," etc. In addition to these basic skills, students will learn the history of classical poetry, drama, and drama courses such as phonological script explanation. In addition, they observe that many drama performances, such as Zhou Xinfang: "four scholars", "Zhao Wuniang", "Han", after "Huarong", "Wen Tianxiang"; the Beijing opera of Ma Lianliang, Tan Fuying and Qiu Shengrong show "in three," lost Jieting "harm", "empty city"; Xun Huisheng's "matchmaker", "Kan Yuzhao"; Mei Lanfang's "Peony Pavilion", "Drunken Beauty", "Farewell to My Concubine", "Luo"; Shang Xiaoyun's "Chinese Princess", "longevity", "Liang Hongyu" mirror; Dean called evolution of wusheng cover "Wu Song" Yu Zhenfei, Zhou Chuanying; opera masters, such as Wang Chuansong's "drunk", "write" and "goudong" qintiao, "the banquet" and the "fifteen Zhejiang Kunsu theatre play through" etc [9].

This comprehensive long term study made the Yiyang cavity system inherit more comprehensively at that time. In this part of the students as the main backbone of the end of 50s, early 60s Jiangxi Jiangxi company adapted a lot of Yiyang traditional opera rehearsal chamber and began to get rid of the past, Yiyang cavity, Luantan, Kunqiang mixed together, and the rehearsal for the Yiyang chamber of new or adapted Jiangxi dramas such as "the resurrection", "Pearl note", "the West Chamber", has a great influence.

In this historical period, the already extinct Yiyang cavern, under the strong intervention of the government, has been comprehensively inherited from many aspects, such as business, drama, performance, music and so on. It can be said that the government made Yiyang cavity "bring back to life".

\section{The Problems of the Government in Promoting the Inheritance and Development of Yiyang Cavity in the Contemporary Era}

Due to some historical reasons, the Gan opera troupe was abolished in 1966. After a very long time, drama activities in Jiangxi were also marginalized or even canceled. Until after the reform and opening up, especially since twenty-first Century, with the government attaches great importance to the traditional opera culture, Yiyang chamber with the troupe began active, the government in the period of protection for Yiyang cavity in many aspects have played a leading role, but also has invested more and more money and manpower. Get a lot of awards, go out to perform a lot, but the Yiyang cavity tune itself does not again brilliant but fell into extinction dilemma, the author thinks that the reason mainly has the following several points:

\subsection{The lack of cultural inheritance in Yiyang}

In the cultural sense, the protection of the local opera is mainly the inheritance of cultural 
relations and the maintenance of the spiritual home. This kind of inheritance has a main body: the inheritor. If there is no inheritor, the opera has been "dead" in a sense or has become a "specimen". The complete heritage is the inheritance of human existence to music [10]. At present the Jiangxi Yiyang heritage chamber only Zou Lili and Zhu Yuexian only a few people, but these people are advanced in age, Yiyang cavity inheritance appeared at the beginning of 1950s, leave no successor. Although the 1959 left Yiyang for still to the cavity, but the opera is a comprehensive art, sing ReadDo play did not fall, not to mention how the western music system based on music to record Chinese traditional opera music?

In May 2016, the national endowment for the arts "Yiyang chamber music talent training plan under the support of Jiangxi theatre actor re-recorded a lot of Yiyang cavity classic tunes, such as" zhuyunfei "and" river water "and" tail made "," step by step Jiao "," cinnamon incense "and" red the coat "," Xiang Luo "," three degree ". Compared to the old artists singing these songs that can now cast according to the concerts in Yiyang chamber of traditional tunes are completely different, a lot of neat rhythm, but a lot less drama charm. In the case of the above, do we ask not to ask if there is no traditional opera or Yiyang opera?

\subsection{The dilemma of the creation of the new opera in Yiyang}

Since 1950s, Jiangxi has appeared some Yiyang opera adaptation or new repertoire, such as "the resurrection", "Pearl", "battle", offering the monument "Yi", "red pearl", which condensed a lot of Yiyang cavity creative performers of hard work and sweat, at the national level are obtained a lot of awards and honors. This undoubtedly enlarges the popularity and influence of the Yiyang cavity, so I think it is very necessary to introduce the high quality repertoire. However, in this historical stage, for the long-term development of Yiyang cavity, what kind of Yiyang opera's top repertoire should we launch for us? Who is the audience for the purpose of the fine drama? Is leadership or fans? What is the goal of the creation of a fine quality repertoire? Is it just to win the prize or to see the people? What is the vitality of these fine plays? After winning the prize, have you survived the market or the audience? The author believes that the so-called "fine quality" should not be created only for the award, but should be the work that can be taken root in the market and loved by the audience. Being popular with audiences doesn't mean vulgar, but winning the so-called boutique doesn't mean elegance. The quality of the play lies in the choice of the market and the audience. Whether the government should recognize and accept the law, living environment and development law of the objective understanding of opera art, not the blind pursuit of "high, big," the blind pursuit of the so-called "fine", this will only make the manpower development delay go wrong, a Yiyang accent.

\subsection{The lack of Yiyang cavity market and the shrinking of the audience}

Since the 2004 Yiyang cavities were identified as the pilot project of China's national folk culture protection project by the Ministry of culture, and Yiyang's cavity was identified as the national intangible cultural heritage in 2006, the government has invested more attention and funds to Yiyang chamber. The new Yiyang Jiangxi cavity staged one after the other awards, one by one, but the Yiyang chamber of the audience is more or less?

Jiangxi province Jiangxi troupe of drummers Wu Wenfei has such a description for the Yiyang chamber performances: "the City show little, go on the show if Luantan play more, not what people love Yiyang cavity."

$\mathrm{Xu}$ Fei, the Yiyang chamber Art Protection Center in Yiyang, has similar description to the performance of Yiyang opera. "Every year there is the task of going to the countryside. Of course, it is playing the playing cavity. You like to listen to it. Yiyang cavity? No one is listening. It is when the leaders come to play and play.

How will the traditional Yiyang cavity, which is divorced from the audience, be developed? Will the Yiyang cavity be alive in the performance project? Or is it always alive at all levels of performance and award? 


\section{Suggestions on the Inheritance and Protection of Yiyang Cavity under the Leadership of the Government}

In reality, the protection and inheritance and development of the Yiyang cavity can not be separated from the government's macro management and the support of human and material resources. But the support of the government should be correct, and the Yiyang cavity can truly regenerate. Based on the discussion of the upper chapter, in view of the problems existing in the management of the government at the present stage, the author puts forward the following suggestions for the inheritance and development of the Yiyang cavity.

\subsection{The reconnaissance of the old artists}

It is the foundation to do well the census work of the old artists in Yiyang cavities, not only to find the students in Nanchang Gan class, but also to explore the old Yiyang entertainers scattered in the folk. During the author write this article, several students in 2015, the national endowment for the arts "Yiyang chamber music training seminar" in Poyang heard a 80 year old folk artists singing a few words of Yiyang traditional opera "riverside" chamber members in a few words, they immediately react, this old man was singing and during the seminar in precious Video - by the end of the Qing Dynasty old artist Li Fudong show "friends" style is very close to the river. Then after understanding that this old artist Li Fudong, Jiang Yongzheng kens disciple, singing is authentic Yiyang accent. Without this seminar, not the accidental discovery, the traditional Yiyang River Riyong Claus cavity may later so gone with him, this is how a thing of shame! Now that the government has the sense of rescue, we can not let the excavating successors still stay at the level of accidental touch, but we should plan, organize and carry out the work in a large scale.

\subsection{The cultivation of the inheritor}

On the basis of the survey of the old artists in Yiyang, it is the cultivation of the new Yiyang inheritors. In the modern, entertainment, diversified, opera this ancient art of survival of the soil has been gradually reduced, while the majority of opera actor dependent troupes benefit is not ideal. In Jiangxi, most of the groups can have economic benefits. In rural areas, and rural people love to listen to the Yiyang chamber less and less. So, in the case of no market in this Yiyang cavity, how can a person without economic benefit be willing to inherit it willingly? This will give the corresponding policy to the government, this group may to do for this ancient opera vector to the corresponding social status, stable income and good development prospects, otherwise is unable to retain talent.

Drama art is a kind of art of time and a kind of live art. It must be transmitted from the previous generation to the next generation, and it will continue to perform, reform and develop on stage. If the inheritance of the inheritor is left, only the cold music and recording and video are left, the activity of this art has been lost. In the face of market is not old tune, the government to help the descendants of most of the training work.

\subsection{The inheritance of the musical style in Yiyang}

There have been a lot of new creative plays in the Yiyang cavity. Whether these new inheritance style and characteristics of Yiyang cavity, which is to be explored. A major feature of the Yiyang chamber is "one sings and prominent percussion accompaniment, chimed in," the history of the Yiyang chamber chimed in mostly by musicians sound rough, chimed in, and as long as the local chimed in to a high of eight degrees singing style is very prominent. But now the creation of the Yiyang chamber of new chimed in sometimes use more voices and sounds, instead of more prominent union. In addition another feature of the Yiyang chamber is not only accompaniment of gongs and drums, music, but a lot of drama now have to use the orchestra's Polyphonic accompaniment, the original phrase between the only percussion accompaniment now plus melody accompaniment [11]. That I once went to a new Jiangxi, not only did not hear the sound of gongs and drums prominent, did not hear the sound of gongs and drums to highlight the weakening, chimed in, chimed in harmony makes the author instantly have the illusion of listening to opera. 
Yiyang cavity can change should also reform, but should be retained in the basic style of music (such as rough, chimed in, outstanding percussion music) on the basis of the reform.

\subsection{Recording the Yiyang cavities and the repertoire}

After a comprehensive inheritance in the 50s of last century, Yiyang chamber will face the danger of being lost because of all kinds of historical and realistic reasons. Although Yiyang continue to produce new cavity, but due to the lack of heritage, Yiyang cavity style has not been passed down. These can sing authentic Yiyang cavity old artist always have to leave one day, once they are gone, leaving only the last century in 50s less recording information and a part of music data. How can Yiyang cavity of this ancient tune by this poor data, which can be handed down? We should organize the old artists who can still sing in Yiyang to carry out full video and recording work in the traditional repertoire. The first recorded some representative excerpts such as: "the river", "friends crazy monk", "Cheng Ying and Qin", "savior send clothing city", "fix the cry", "black basin Tianshan Ji", "the biography" etc.

\section{Conclusion}

The advantage of Yiyang led by the government is that the top-down government behavior has played the role of Yiyang's cavity in some specific historical periods, for example, Gan's class's inheritance to Yiyang chamber. It is disadvantageous in these government behavior has led to the Yiyang chamber of non-market non folk heritage, the Yiyang chamber will once again lost, also led to the Yiyang chamber of "a new boutique repertoire" just need to "on" government without the need for "under the" responsible for the market and the audience, or say, Yiyang chamber now has basically no market audience. This requires the government combined with the actual situation in the census, the old artist, culture heritage and the old artists place Yiyang cavity style, comprehensive recording and marketing to increase efforts to the vanishing old opera left to save data and continue to study the space of survival and development.

\section{References}

[1] Wang M. Organization and Analysis of Study Works on Traditional Opera and Thoughts at the Beginning of 21 (st) Century[J]. Studies in Culture \& Art, 2016.

[2] Ma G. On the Classification of Traditional Operas and Levels of Various Operas[J]. Drama, 2016.

[3] Li H, Cai H. "Think Down Thousands of Years" and "Keep Pace with the Times" Traditional Opera Reconstruction in the Early of New China[J]. Contemporary China History Studies, 2016.

[4] Wang X. The Dance Characters Performing in the Perspective of Form and Spirit Concept of Chinese Traditional Operas[J]. Journal of Beijing Dance Academy, 2017.

[5] Yong-Chun Y U, University M N. Conflict Between the Shape of Traditional Operas and the Spirit of Modern Dramas: The Relationship Between Guo Moruo's Historical Dramas and Operas[J]. Journal of Guangdong Polytechnic Normal University, 2016.

[6] Yang S, Zhong X. Research on the Tourism Exploitation of Traditional Opera,Intangible Culture Heritage in Guangxi[J]. Journal of Guilin Normal College, 2016.

[7] Xu Y. On the Stylistic Construction of Wang Guowei's History of Traditional Opera in the Song and Yuan Dynasties[J]. Theoretical Studies in Literature \& Art, 2016.

[8] Zi-Fang X U. Northern Opera Sang in Southern Style and Kun Style:Thinking over Opera Form Evolvement of Yuan and Ming Theatre[J]. Hundred Schools in Arts, 2013.

[9] Wei J, University A H X. The Influence of Opera Performance on the Creation of Popular 
Woodblock Prints in the Late Ming Dynasty[J]. Ethnic Art Studies, 2016.

[10] Wang L C. A Restricted View of the Style and Features of The Story of the Lute from the Scripts Published in Ming \& Qing Dynasties[J]. Journal of Liaocheng University, 2012.

[11] Wan-Xia L I. Exploring the Causes of Foshan Becoming Important Town of Local Opera Performances in Late Ming and Early Qing Dynasties[J]. Journal of Foshan University, 2016. 